\title{
SYNTHESIS AND CHARACTERIZATION OF HYDROXYPROPYL CELLULOSE-CYSTEAMINE CONJUGATE AS A NOVEL CATIONIC THIOMER WITH LIPOPHILIC PROPERTIES
}

\section{DENI RAHMAT*, FIKRY A. R. RAHMAN, LILIEK NURHIDAYATI, DIAN RATIH LAKSMITAWATI}

\author{
Faculty of Pharmacy, Pancasila University, Srengseng Sawah, Jagakarsa, Jakarta Selatan, 12640, Indonesia
}

Email: denrerum@gmail.com

Received: 28 Sep 2018, Revised and Accepted: 12 Dec 2018

\section{ABSTRACT}

Objective: Thiomers have been known as polymer with mucoadhesive properties. The aim of this study was to synthesize the mucoadhesive potential of hydroxypropyl cellulose-cysteamine conjugate (HPC-cysteamine).

Methods: The parent polymer HPC was chemically modified by introducing sulphydryl bearing compound using reductive amination. HPCcysteamine conjugates were prepared at reaction $\mathrm{pH}$ value of 5 . The reaction was stabilized by the addition of cyanoborohydride. Afterwards, the conjugate was evaluated for optimum free thiol group, swelling behavior, viscosity and mucoadhesive properties.

Results: The conjugates showed maximum thiol incorporation on HPC of $1063.03 \pm 64.27 \mu$ mol/g. The disulphide groups content was $278.71 \pm 32.14$ $\mu \mathrm{mol} / \mathrm{g}$. Mucoadhesion studies revealed that mucoadhesion of HPC-cysteamine demonstrated 26 h. The swelling behaviour of HPC-cysteamine tablets increased within the time period of study. The viscosity of HPC-cysteamine was higher than that of unmodified HPC. The thermal profile of HPC-cysteamine and unmodified HPC analyzed by differential scanning calorimetry (DSC) displayed a different enthalpy ( $\Delta \mathrm{H}$ ) value.

Conclusion: HPC-cysteamine conjugate renders better properties which might be more beneficial for drug delivery system compared to unmodified HPC.

Keywords: Hydroxypropyl cellulose, Cysteamine, Thiomer, Mucoadhesive, Swelling

(C) 2019 The Authors. Published by Innovare Academic Sciences Pvt Ltd. This is an open access article under the CC BY license (http://creativecommons.org/licenses/by/4.0/) DOI: http://dx.doi.org/10.22159/ijap.2019v11i1.30014

\section{INTRODUCTION}

The development of multifunctional polymers in pharmaceutical technology has been influenced by various factors, including biocompatible and biodegradable properties. Thiomers are polymers modified by introducing thiol groups on the structure of the polymer backbone by chemical reactions. They are preferred for improving bioavailability of drugs and a fascinating choice for oral drug delivery. The less permeable drugs can be formulated with thiomers in order to improve their absorption through mucosal membranes due to prolonged resident time and opening tight junctions $[1,2]$.

The administration of macromolecule drugs such as protein drugs is required in several diseases. However, their effectiveness is still poor due to low bioavailability thus the application of thiolated polymers might be beneficial to overcome the problems in their delivery. These multifunctional polymers demonstrate some advantages in mucoadhesion and permeation enhancing properties compared to unmodified polymers $[3,4]$.

Cellulose derivates with their properties such as inert, biocompatibility, water solubility and stability, render challenges in improving their mucoadhesive and permeation enhancing properties. Hydroxyethyl cellulose (HEC) has been conjugated with cysteamine to immobilize free thiol groups by oxidation and reductive amination. The resulting thiomer is a novel cationic thiomer that shows substantially improved mucoadhesive properties and soluble at a broad range of $\mathrm{pH}$ value. In addition, ionic gelation method is applicable to generate HEC-cysteamine based nanoparticles which have further improved the permeation enhancing properties [5-7].

On the one hand, HEC contains several-OH groups that can be chemically modified. On the other hand, hydroxypropyl cellulose (HPC) with few-OH groups in repeating sugar units hydroxypropylated using propylene oxide confers it lipophilic properties [8]. It was therefore the aim of this study to chemically modified HPC with cysteamine in order to provide various properties of the cationic thiomers.

\section{MATERIALS AND METHODS}

\section{Materials}

Cysteamine. $\mathrm{HCl}$, sodium periodate, ethylene glycol, sodium cyanoborohydride, 2-(N-morpholino) ethanesulfonic acid (MES hydrate), trinitro-benzensulfonic acid (TNBS), sodium borohydride, dialysis tubing cellulose membrane (molecular weight cut-off of 12 $\mathrm{kDa})$, HPC ( $75-150 \mathrm{mPa}$. S, $5 \%$ in $\mathrm{H}_{2} \mathrm{O}$ at $20{ }^{\circ} \mathrm{C}$, molecular weight: 100.000) were purchased from Sigma-Aldrich, Singapore. Mucus was collected from porcine intestine which was obtained freshly from the slaughter house, Bekasi city, West Java, Indonesia.

\section{Synthesis of oxidized HPC}

The covalent attachment of cysteamine to HPC was achieved by the formation of amide bonds between the amine group of cysteamine and aldehyde groups of oxidized HPC. HPC was oxidized by sodium periodate as described in the literature. Briefly, $1.5 \mathrm{~g} \mathrm{HPC}$ was dissolved in the mixture of $20 \mathrm{ml}$ of ethanol and $120 \mathrm{ml}$ of demineralized water in $500 \mathrm{ml}$ erlenmeyer flask wrapped with aluminium foil. Afterwards, $10 \mathrm{ml}$ of $8 \%(\mathrm{w} / \mathrm{v})$ sodium periodate was added to the solution. The reaction mixture was incubated for 2 $\mathrm{h}$ under permanent stirring. $200 \mu \mathrm{l}$ of ethylene glycol was added to inactivate any unreacted periodate. The reaction was stirred for $1 \mathrm{~h}$ at room temperature and then dialyzed exhaustively (molecular weight cut-off of $12 \mathrm{kDa}$; dialysis tubing cellulose membrane) against water for $3 \mathrm{~d}$. The water was changed at least three times a day. The purified product was freeze dried $\left(-78{ }^{\circ} \mathrm{C}, 0.01 \mathrm{mbar}\right.$, VirTis, Gardiner, ME) and kept at $4{ }^{\circ} \mathrm{C}[6,7]$.

\section{Synthesis of HPC-cysteamine conjugates}

Synthesis of HPC-cysteamine conjugates were synthesized according to a method described previously. Oxidized HPC was dissolved in $100 \mathrm{ml}$ of $0.1 \mathrm{M}$ MES buffer at a final concentration of $1 \%(\mathrm{w} / \mathrm{v})$ and cysteamine. $\mathrm{HCl}$ was added to the solution at various concentrations $(0.25 ; 0,5 ; 1 \% \mathrm{w} / \mathrm{v})$. The mixture was adjusted to $\mathrm{pH} 5.0$ and $\mathrm{NaCNBH}_{3}$ served as reductor was added at a concentration of $8 \%$ $(\mathrm{w} / \mathrm{v})$. The reaction was conducted for $72 \mathrm{~h}$ at room temperature. Afterwards, the reaction mixture was exhaustively dialyzed one time against distilled water, one time against $0.2 \mathrm{mmol} \mathrm{HCl}$, then two times against the same medium but containing $1 \%(\mathrm{w} / \mathrm{v}) \mathrm{NaCl}$ in tubing (molecular weight cut-off of $12 \mathrm{kDa}$; dialysis tubing cellulose membrane) at $10{ }^{\circ} \mathrm{C}$ in the dark followed by lyophilization $\left(-78{ }^{\circ} \mathrm{C}\right.$ 0.01 mbar, VirTis, Gardiner, ME). Control polymers were prepared in exactly the same way without the addition of $\mathrm{NaCNBH}_{3}$ during the reaction $[6,7]$. 


\section{Evaluation of the thiol group content}

The content of free sulfhydryl groups immobilized on the polymer backbone was measured using Ellman's reagent. Thereby, the amount of cysteamine bound to HPC was calculated from a corresponding calibration curve. In addition, the amount of disulfide groups on HPC-cysteamine conjugate formed due to the exposure of oxygen during the process of reductive amination was determined after reduction with $\mathrm{NaBH}_{4}$. The disulfide content on HPCcysteamine conjugate was obtained by subtracting the amount of thiol groups before the reduction from the total amount of thiol groups [9].

\section{Fourier transporm infrared spectroscopy (FTIR) studies}

FTIR spectra were performed by BRUKER Vertex 70 spectrometer with aMIRacle ATR-diamond unit (Attenuated Total Reflection) in the range $500-4000 \mathrm{~cm}^{-1}$. The instrument displays a spectral resolution of $\sim 4 \mathrm{~cm}^{-1}$. All of the samples were pressed onto the diamond window by a plane steel cone [6]

\section{Viscosity measurement}

Rheological studies were carried out by mixing $4 \mathrm{~g}$ of porcine mucin with $25 \mathrm{ml}$ of demineralized water with continuous stirring. Afterwards, the $\mathrm{pH}$ of the mixture was adjusted to 6.8 and diluted to a final concentration of $8 \%(\mathrm{~m} / \mathrm{v})$ with $0.2 \mathrm{M}$ phosphate buffer $\mathrm{pH}$ 6.8. Unmodified HPC, oxidized HPC and HPC-cysteamine, respectively, were solubilized in demineralized water to get a concentration of $6 \%(\mathrm{~m} / \mathrm{v})$ and were mixed homogeneously with an equal volume of $8 \%(\mathrm{~m} / \mathrm{v})$ mucin solution. Solutions containing either $3 \%(\mathrm{~m} / \mathrm{v})$ of the polymer or $4 \%(\mathrm{~m} / \mathrm{v})$ mucin in $50 \mathrm{mmol}$ phosphate buffer $\mathrm{pH} 6.8$ were used as references. Viscosity studies were performed using viscometer Brookfiel RV at $100 \mathrm{rpm}$ [10].

\section{Evaluation of the swelling behavior}

Solid form of unmodified HPC, oxidized HPC, and HPC-cysteamine were compressed into tablet with $50 \mathrm{mg}$ in weight and $5.0 \mathrm{~mm}$ in diameter. Single punch of tablet press machine with force of $10 \mathrm{kN}$ was used for the preparation of tablets. The amount of absorbed water in the tablets was performed by a gravimetric method. Thus, $50 \mathrm{mg}$ tablets of unmodified HPC, oxidized HPC, HPC-cysteamine were fixed on a needle and submersed in a test tube containing 0.1 $\mathrm{M}$ phosphate buffer solution, $\mathrm{pH} 6.5$ at $37 \pm 0.5{ }^{\circ} \mathrm{C}$. At predetermined time points the test tablets were taken out of the buffered medium and weighted. The amount of water uptake was measured gravimetrically. The swelling ratio was then determined according to the following equation:

$$
\text { Swelling ratio }=\frac{W u t}{W o}
$$

where Wut is the weight of uptaken water at time $t$ and Wo is the initial weight of the dry tablet [11].

\section{In vitro mucoadhesion studies}

Unmodified HPC, oxidized HPC and HPC-cysteamine tablets of $50 \mathrm{mg}$ were attached to a freshly excised intestinal porcine mucosa, which was fixed on the cylinder (diameter: $4.4 \mathrm{~cm}$; height $5.1 \mathrm{~cm}$; apparatus 4-cylinder, USP). Afterwards, the cylinder was put in the tablet dissolution testing instrument with $500 \mathrm{ml}$ of $100 \mathrm{mmol}$ phosphate buffer $\mathrm{pH} 6.8$ at $37{ }^{\circ} \mathrm{C}$ and agitated with $100 \mathrm{rpm}$. The time required for the test tablets to detach was recorded [10].

\section{Solubility test}

Solubility test was performed to examine the effect of the changes of $\mathrm{pH}$ value on the aggregation of the polymer solution. Briefly, $0.5 \mathrm{~g}$ of HPC-cysteamine was solubilized in $50 \mathrm{ml}$ of distilled water and the $\mathrm{pH}$ of the solution was changed in the range of 1-14 by adding $\mathrm{HCl}$ or $\mathrm{NaOH}$ solution.

\section{Differential scanning calorimetric (DSC) analysis of unmodified HPC and HPC-cysteamine}

The DSC patterns of unmodified HPC and HPC-cysteamine were investigated using a PerkinElmer Thermal Analysis at a scanning rate of $10^{\circ} \mathrm{C} / \mathrm{min}$ from 20 to $300^{\circ} \mathrm{C}$ under nitrogen gas stream at a flow rate of $40 \mathrm{ml} / \mathrm{min}$. Unmodified HPC and HPC-cysteamine of 3-5 mg were precisely weighed and put into flat-bottomed aluminum pans [12].

\section{Statistical data analysis}

The results are given in the mean $\pm S D(n=3)$. Statistical data analysis was generated using the student t-test, two tails with $95 \%$ confidence interval ( $p$-value $<0.05)$ as the minimal level of significance.

\section{RESULTS AND DISCUSSION}

\section{Synthesis of oxidized HPC}

Unmodified HPC was reacted with sodium periodate to obtain oxidized HPC. The viscosity of the reaction mixture decreased after the reaction due to the opening of the glucose rings. The degree of oxidation of oxidized HPC was influenced by sodium periodate concentration. Increase in sodium periodate concentration results in an increase in the degree of oxidation. Unmodified HPC requires a periodate ion for the oxidation. The I-O bonds of the periodate will break the glucose ring by attacking hydroxyl groups of the vicinal diols of HPC $[13,14]$.

\section{Synthesis of HPC-cysteamine conjugates}

The reductive amination of oxidized HPC involved sodium cyanoborohydride as reductor and cysteamine as ligand with a thiol group to generate HPC-cysteamine conjugate. As reported in previous studies, the stable conjugation of cysteamine took place at $\mathrm{pH}$ value of 5 . The stable conjugation of secondary amine was achieved in the presence of sodium cyanoborohydride. Since thiomers are constructed by immobilization of ligands with sulfhydryl groups on the backbone of polymers, the resulting aldehydic groups on the oxidized HPC were reacted with primary amino groups of cysteamine to form cationic thiomer $[6,15]$. In contrast, free thiol groups were not found in a control polymer.

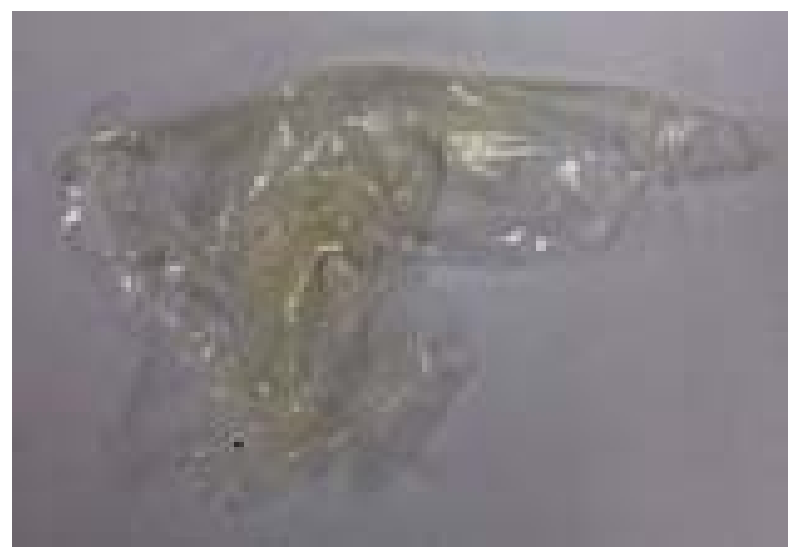

Fig. 1: HPC-cysteamine

\section{Evaluation of the thiol group content}

After the freeze drying process, HPC-cysteamine displayed a transparent film as shown in fig. 1 and was soluble in aqueous solutions with a broad range of $\mathrm{pH}$ value. The use of highest concentration of cysteamine. $\mathrm{HCl}$ resulted in the highest amount of free thiol group $(1063.03 \pm 64.27 \mu \mathrm{mol} / \mathrm{g})$ as shown in table 1. During the reaction, oxidation occurred to produce disulfide bonds on HPCcysteamine conjugate. The determination of disulfide bonds content required reduction process of HPC-cysteamine conjugate to obtain the additional of free thiol group. Hence, after the reduction total free thiol group was measured by the addition of Ellman's reagent [9]. It was found that the amount of disulfide bonds on the conjugate with the highest amount of free thiol groups was $278.71 \pm 32.14$ $\mu \mathrm{mol} / \mathrm{g}$. 
Table 1: Degree of thiolation of HPC-cysteamine

\begin{tabular}{ll}
\hline Amount of cysteamine. $\mathrm{HCl}(\%)$ & Amount of free thiol group $(\boldsymbol{\mu m o l} / \mathbf{g})^{*}$ \\
\hline 0.25 & $210.35 \pm 90$ \\
0.5 & $637.9143 \pm 363.85$ \\
1.0 & $1063.03 \pm 64.27$ \\
\hline
\end{tabular}

Notes: *: Data represented as mean \pm SD $(n=3)$

\section{FTIR studies}

The spectra of HPC-cysteamine as shown in fig. 2 in the range of $800-2300 \mathrm{~cm}^{-1}$ and around $2500 \mathrm{~cm}^{-1}$ displays comparatively flat as shown by that of cysteamine. This could indicate the presence of $\mathrm{S}-\mathrm{H}$ in HPC-cysteamine conjugate. In addition, HPC-cysteamine shows broader spectra compared to unmodified HPC and oxidized HPC in the range of $3310-3500 \mathrm{~cm}^{-1}$ and of $2500-2900 \mathrm{~cm}^{-1}$ to demonstrate the the presence of secondary amine and free thiol groups, respectively as results of the conjugation [16].

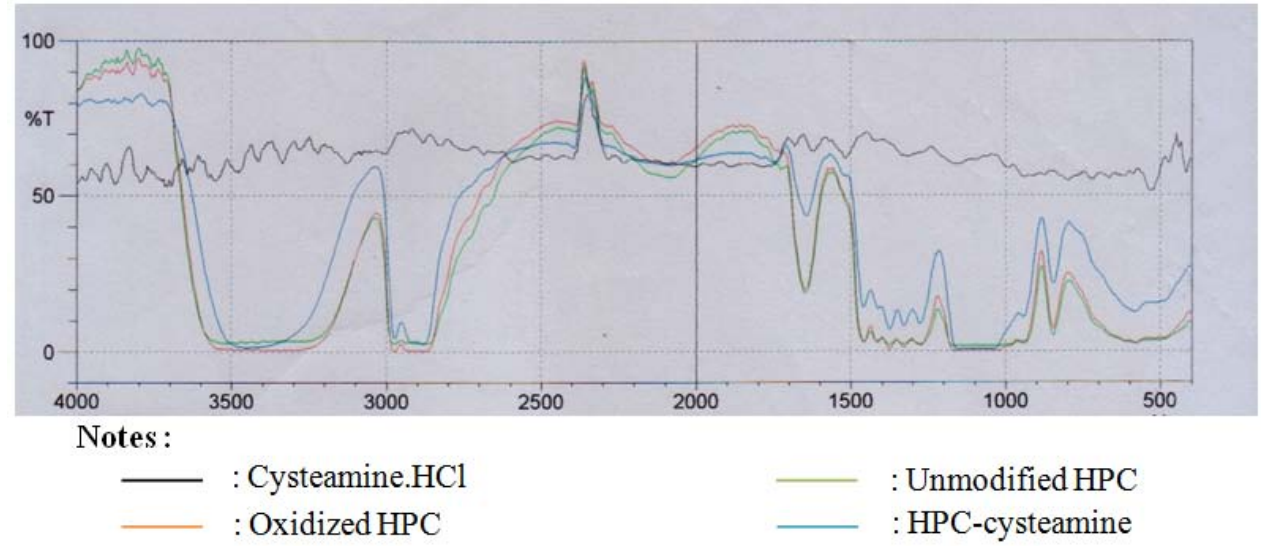

Fig. 2: FTIR of the test polymers

\section{Viscosity measurement}

Unmodified HPC 3\% (m/v) demonstrated the lowest viscosity compared to oxidized HPC and HPC-cysteamine as shown in table 2 due to low solubility of unmodified HPC in aqueous solution. However, after the addition of mucin $4 \%(\mathrm{~m} / \mathrm{v})$ the viscosity of HPCcysteamine conjugate $3 \%(\mathrm{~m} / \mathrm{v})$ was nearly 3 -fold higher compared to its initial value while that of oxidized HPC 1.8-fold. Since no thiol groups immobilized on oxidized HPC, the viscosity of the mixture of oxidized HPC and mucin was theoritical additive value. The free thiol groups of HPC-cysteamine and the cysteine-rich subdomaines of the mucus form disulfide bonds. Unmodified HPC seems to swell significantly after mixing with mucin leading to the increase in its viscosity which was 4.7 -fold higher compared to its initial value. The mucin could support the swelling of unmodified HPC thus their noncovalent interactions could lead to the increase in viscosity. Hence, lipophilic properties of unmodified HPC and HPC-cysteamine might play an important role that they could have better capability of penetrating into the mucus gel compared to HEC and other cellulose derivates. In case of unmodified HEC, it was reported that the viscosity of unmodified HEC remains unchanged after mixing with the mucin.

Table 2: The effect of mucin on viscosity

\begin{tabular}{ll}
\hline Polymer & Viscosity (cps)* \\
\hline Unmodified HPC & $137.33 \pm 7.51$ \\
Oxidized HPC & $251.00 \pm 10.15$ \\
HPC-cysteamine & $256.33 \pm 15.50$ \\
Unmodified HPC+mucin & $646.67 \pm 6.51$ \\
Oxidized HPC+mucin & $443.33 \pm 10.07$ \\
HPC-cysteamine+mucin & $738.00 \pm 16.00$ \\
Mucin & $215.33 \pm 8.50$ \\
\hline
\end{tabular}

Notes: ${ }^{*}$ :Data represented as mean \pm SD $(n=3)$

\section{Evaluation of the swelling behavior}

The thiol groups are able to influence the cohesiveness of tablets shown by their capability of swelling. Unmodified HPC, oxidized HPC and HPC-cysteamine tablets undertook swelling in the medium. The weight of unmodified HPC and oxidized HPC tablets increased within $120 \mathrm{~min}$ and then decreased slowly as shown for its swelling ratio in table 3. In contrast, the increase in weight of HPC-cysteamine tablets remained stable within $300 \mathrm{~min}$ due to their cohesive properties as disulfide bonds were formed in the tablets. The immobilization of thiol groups on HPC along with its lipophilic properties interfered significantly with the integrity and stability of the tablet. However, unmodified HPC and oxidized HPC tablets seem to disintegrate and erode slowly as the absence of free thiol groups and disulfide bonds. 
Table 3: Swelling behaviour of the test tablets

\begin{tabular}{llll}
\hline Time (min) & Swelling ratio $^{*}$ & & \\
\cline { 2 - 3 } & Unmodified HPC & Oxidized HPC & HPC-cysteamine \\
\hline 15 & $1.038 \pm 0.01$ & $1.0331 \pm 0.004$ & $1.0432 \pm 0.001$ \\
60 & $1.042 \pm 0.01$ & $1.0391 \pm 0.01$ & $1.0651 \pm 0.002$ \\
120 & $1.058 \pm 0.007$ & $1.0480 \pm 0.003$ & $1.0748 \pm 0.005$ \\
180 & $1.055 \pm 0.0001$ & $1.0447 \pm 0.006$ & $1.0807 \pm 0.0003$ \\
240 & $1.050 \pm 0.0001$ & $1.0342 \pm 0.014$ & $1.0912 \pm 0.001$ \\
300 & $1.046 \pm 0.0004$ & $1.0272 \pm 0.0124$ & \\
\hline
\end{tabular}

Notes: *: Data represented as mean $\pm \mathrm{SD}(\mathrm{n}=3)$

\section{In vitro mucoadhesion studies}

Mucoadhesion study of unmodified HPC, oxidized HPC and HPCcysteamine were carried out using the rotating cylinder. The attachment duration of HPC-cysteamine tablets was $26 \mathrm{~h}$ whereas that of unmodified HPC and oxidized HPC were $2 \mathrm{~h}$. It demonstrated that reductive amination prolongs the mucoadhesive properties as the free thiol groups of HPC-cysteamine and the cysteine-rich subdomaines of the mucus interacted to form disulfide bonds. Due to lipophilic properties, swelling behavior of HPC-cysteamine involved optimally in the interdiffusion process between HPC-cysteamine and the mucus gel. Thereby, HPC-cysteamine tablets was able to attach significantly. The oxidation of the conjugate could take place resulting in the formation of disulfide bonds during the experiments but free thiol groups was still present in HPC-cysteamine tablet. Hence, HPC-cysteamine tablet could swell to penetrate into the mucus gel [17].

\section{Solubility test}

Unmodified HPC can be solubilized in the mixture of distilled water and ethanol to form clear aqueous gels whereas HPC-cysteamine is soluble in distilled water. The formation of disulfide bonds lowers the solubility of HPC-cysteamine in distilled water. In addition, the solubility of the conjugate was not influenced by acidic and basic $\mathrm{pH}$ of the solution. The precipitation did not occur when $1 \mathrm{M} \mathrm{HCl}$ or $1 \mathrm{M}$ $\mathrm{NaOH}$ was added to the solution.

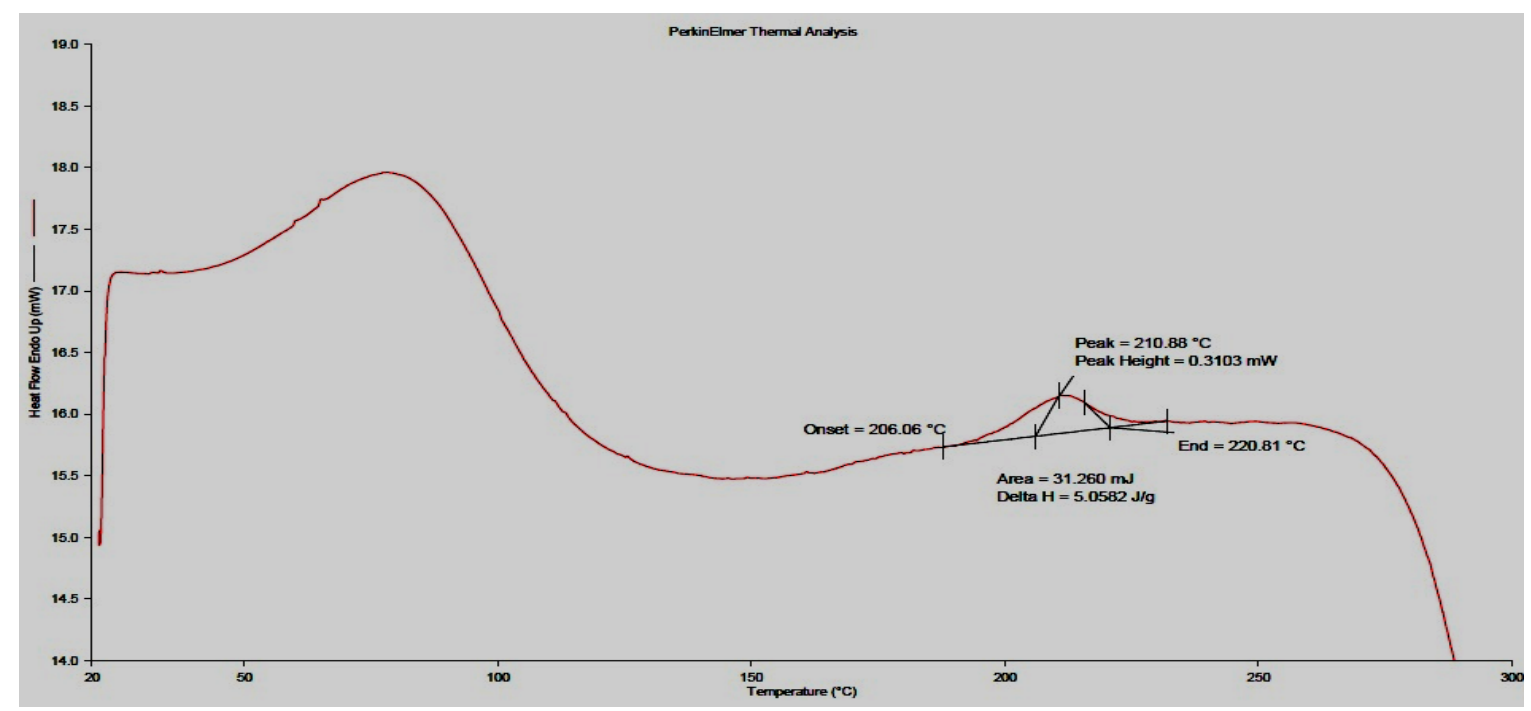

Fig. 3: DSC thermal analysis of unmodified HPC

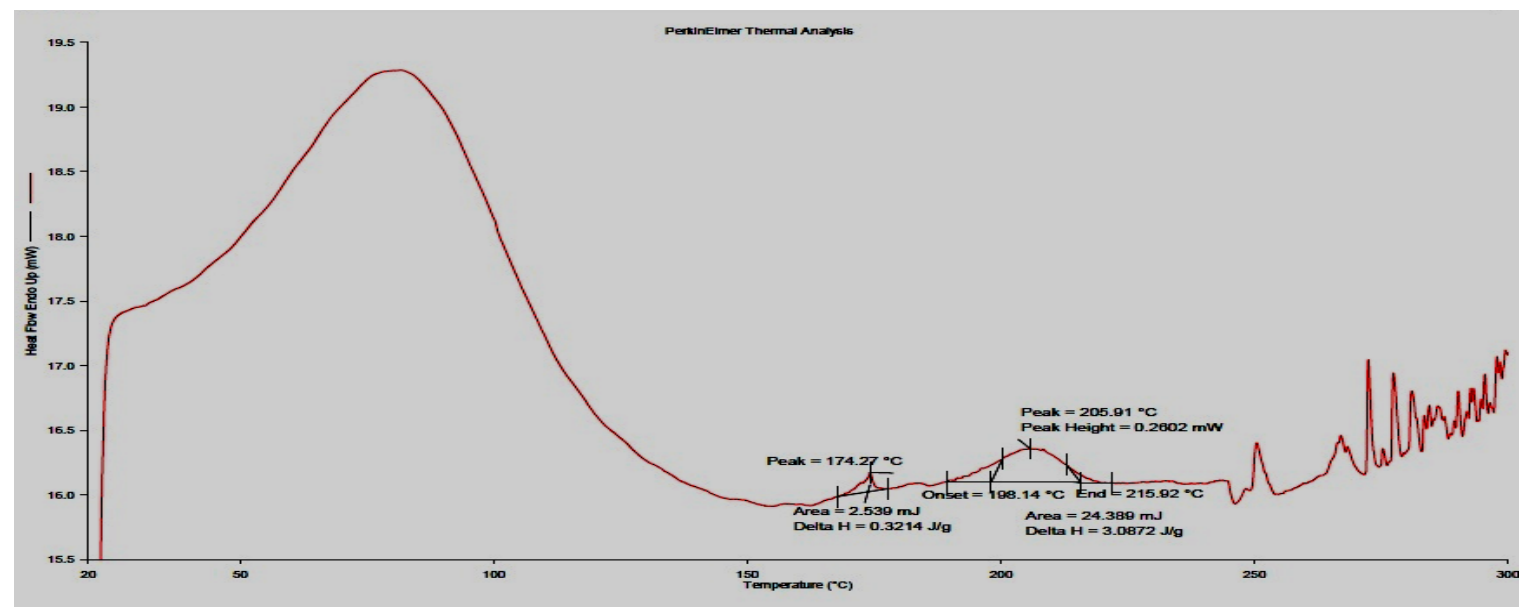

Fig. 4: DSC thermal analysis of HPC-cysteamine 


\section{DSC analysis of unmodified HPC and HPC-cysteamine}

Based on the exothermic peak as depicted in fig. 3 and 4 , the change in enthalpy $(\Delta \mathrm{H})$ of unmodified HPC is $5.08282 \mathrm{~J} / \mathrm{g}$ while that of HPC-cysteamine is $3.0872 \mathrm{~J} / \mathrm{g}$ as shown in fig. 3 and fig. 4 respectively. The decrease in $\Delta \mathrm{H}$ will affect the solubility of the polymer thus HPC-cysteamine was more soluble in distilled water than unmodified HPC. The increase in solubility is in accordance with the decrease in the melting point [18]. Thereby, the test polymers are showing different thermal behaviors. The oxidation and reductive amination process did not change a lot in the melting point. Therefore, lipophilic properties of the parent polymer could still exist in the conjugate.

\section{CONCLUSION}

HPC-cysteamine is a novel cationic thiomer with lipophilic properties that could influence its swelling behavior and mucoadhesive properties. The interpenetration between HPCcysteamine and the mucus could be intense. Hence, the diffusion of free drugs dispersed in the matrix of HPC-cysteamine through the mucus might be deeper compared to in the matrix of unmodified HPC. Moreover, HPC-cysteamine based nanoparticles could be absorbed from the entire gastrointestinal tract with a different mucosal integrity.

\section{ACKNOWLEDMENT}

The authors gratefully acknowledge financial support from Ristek DIKTI Indonesia (Penelitian Berbasis Kompetensi).

\section{AUTHORS CONTRIBUTIONS}

All the author have contributed equally

\section{CONFLICT OF INTERESTS}

The author reports no declarations of interest

\section{REFERENCES}

1. Karolewicz B. A review of polymers as multifunctional excipients in drug dosage form technology. Saudi Pharm J 2016;24:525-36.

2. Rao MRT, Gaikwad SR, Shevate PM. Synthesis and characterization of a novel mucoadhesive derivative of psyllium seed polysaccharide. Int J Pharm Pharm Sci 2017;9:166-75.

3. Colombo P, Sonvico F, Colombo G, Bettini R. Novel platforms for oral drug delivery. Pharm Res 2009;26:601-11.

4. Roy S, Prabhakar B. Bioadhesive polymeric platforms for transmucosal drug delivery systems-a review. Trop J Pharm Res 2010;9:91-104.
5. Roy DS, Rohera BD. Comparative evaluation of rate of hydration and matrix erosion of HEC and HPC and study of drug release from their matrices. Eur J Pharm Sci 2002;16:1939.

6. Rahmat D, Sakloetsakun D, Shahnaz G, Perera G, Kaindl R, Bernkop-Schnürch A. Design and synthesis a novel cationic thiomer. Int J Pharm 2011;411:10-7.

7. Rahmat D, Muller C, Barthelmes J, Shahnaz G, Martien R, Bernkop-Schnürch A. Thiolated hydroxyethyl cellulose: design and in vitro evaluation of mucoadhesive and permeation enhancing nanoparticles. Eur J Pharm Biopharm 2013;83:149. 55.

8. Trivedi MK, Nayak G, Patil S, Tallapragada RM, Mishra R. Influence of biofield treatment on physicochemical properties of hydroxyethyl cellulose and hydroxypropyl cellulose. J Mol Pharm Org Process Res 2015;3:1-7.

9. Hombach J, Palmberger TF, Bernkop Schnurch A. Development and in vitro evaluation of a mucoadhesive vaginal delivery system for nystatin. J Pharm Sci 2009;98:555-64.

10. Bernkop Schnurch A, Hornof M, Zoidl T. Thiolated polymerthiomers: synthesis and in vitro evaluation of chitosan-2iminothiolane conjugates. Int J Pharm 2003;260:229-37.

11. Kast CE, Bernkop Schnurch A. Thiolated polymers-thiomers: development and in vitro evaluation of chitosan-thioglycolic acids conjugates. Biomaterials 2001;22:2345-52.

12. Kar K, Pal RN, Bala NN. Preparation, characterisation and evaluation of ropinirole hydrochloride loaded controlled release microspheres using solvent evaporation technique. Int J Pharm Pharm Sci 2018;10:57-67.

13. Sussich F, Cesaro A. The kinetics of periodate oxidation of carbohydrates: a calorimetric approach. Carbohydr Res 2000;329:87-95.

14. Le-Tien C, Millette M, Lacroix M. Modified alginate matrice for the immobilization of bioactive agents. Biotechnol Appl Biochem 2004;38:98-107.

15. Tadanier J, Hallas R, Martin JR, Stanaszek RS. Observation relevant to the mechanism of the reductive aminations of ketones with sodium cyanoborohydride and ammonium acetate. Tetrahedron 1981;37:1309-16.

16. Koranyi TI, Moreau F, Rozanov VV, Rozanova EA. Identification of SH groups in zeolite-supported HDS catalysts by FTIR spectroscopy. J Mol Struct 1997;410:103-10.

17. Bernkop Schnurch A. Thiomers: a new generation of mucoadhesive polymers. Adv Drug Delivery Rev 2005;57:156982.

18. Muddukrishna BS, Dengale SJ, Shenoy GG, Bhat K. Preparation, solid state characterisation of paclitaxel and naringen cocrystals with improved solubility. Int J Appl Pharm 2016;6:32-7. 